\title{
ACTIVE CONTOUR AND LEVEL SET USING SEGMENTATION IN MEDICAL IMAGES
}

\author{
Disha Bawa \\ Department of CSE \\ Sutton, Surrey, United Kingdom
}

\begin{abstract}
Image processing is a method to perform some methods on the image to get enhanced image and get some useful information from the image. Segmentation of the image refers to extraction of important information from the specific region of the image. There are two methods used for segmentation of the image i.e. Level set function and active contour method. The level set function is used to define the outline of the object and active contour is boundary based segmentation of different medical image. Active contour is used in CT images, MRI and cardiac images and different regions of human body
\end{abstract}

Keywords-Segmentation, Active Contour, Level Set, Image Processing

\section{INTRODUCTION}

The basic idea is to start with initial boundary shapes represented in a form of closed curves, i.e. contours, and iteratively modify them by applying shrink/expansion operations according to the constraints of the image. Those shrink/expansion operations, called contour evolution, are performed by the minimization of an energy function like traditional region-based segmentation methods. Snake id perhaps the classical model, if you are segmenting a unique object from an initial contour, it's generally faster especially for a single objects. If you have multiple objects and don't really know where, level sets will be more efficient, since they usually care less about the initialization, and they can split automatically to detect more than one connected component.

Active contours, or snakes, are computer-generated curves that move within images to find object boundaries (note that the 3D version is often known as deformable models or active surfaces in the literature). They are often used in computer vision and image analysis to detect and locate objects, and to describe their shape. For example, a snake might be used to automatically find a manufactured part on an assembly line; one might be used to find the outline of an organ in a medical image

\section{METHOD}

\section{A. Level set function}

The level set function is a way to represent a known outline of an object by setting the pixels inside the outline to positive values, the pixels on the outside to negative values and the

\author{
Aditya Sharma \\ Department of CSE \\ IKGPTU, Kapurthala, Punjab, India
}

pixels around the outline to 0 . Thus the zero level of the level set function is where the outline is found. Level set is also a contour model based on curve evolution.

\section{Level-set methods}

Level-set methods provide a very effective framework for numerical explanation of curves and surfaces and therefore are widely appropriate in many areas including computational fluid dynamics problems as well as image processing and computer vision applications. The principal idea behind levelset methods is to avoid explicit parametric representation of geometrical objects such as curves or surfaces, and instead represent these objects implicitly in terms of a function defined on a fixed computational grid. Contrary to explicit contour representations, level-set methods are also successful in capturing topological changes of objects. For example, level-set can easily handle splitting of a connected region into two or more disjoint parts.

Curves and surfaces can be described implicitly as the zero level-sets of some sufficiently smooth function $\phi$ :

$\mathcal{C}=\{\mathrm{x} \in \Omega: \phi(\mathrm{x})=0\}$

Here $\mathbf{x}$ denotes a point in a region $\Omega$.

\section{Level-sets for segmentation}

Proposed algorithm, which has since been widely used for different image segmentation tasks including medical images. It is a special case of the Mumford-Shah optimal partition and approximation problem designed for binary images. However, it also gives very good results in case of gray-scale and vectorvalued (e.g., RGB) images. Next, we briefly review the method. Suppose we are given a domain $\Omega$ divided by a contour $\Gamma=\{\phi(\mathbf{x})=0\}$ into two (possibly unconnected) subregions $\Omega_{\text {in }}=\{\phi(\mathbf{x})>0\} \quad$ and $\quad \Omega_{\text {out }}=\{\phi(\mathbf{x})<0\}$. The function $\phi$ is a level-set function defining the segmenting contour. Let $I(\mathbf{x})$ be an image defined on the region $\Omega$. The method relies on the minimization of an intensity-based energy functional given by:

$E\left(\phi, c_{1}, c_{2}\right)=|\Gamma|+\int_{\Omega \text { in }}\left|I(\mathrm{x})-c_{1}\right|^{2} \mathrm{dx}+\int_{\Omega \text { out }}\left|I(\mathrm{x})-c_{2}\right|^{2} \mathrm{dx}$, 
(2)

where $|\Gamma|$ is a length of the segmenting contour, and $c_{1}$ and $c_{2}$ denote average intensities inside and outside of the segmenting contour $\Gamma$, respectively, in the following way:

$\mathrm{c} 1=1|\Omega \operatorname{in}||\Omega \operatorname{inI}(\mathbf{x}) \mathrm{d} \mathbf{x}, \mathrm{c} 2=1| \Omega$ out $\mid \int \Omega \operatorname{out}(\mathbf{x}) \mathrm{d} \mathbf{x}$.

(3)

Hence, the functional $E$ given in (2) penalizes local discrepancy from the average intensity in the segmented regions. Using the gradient flow method, the regularized minimization problem can be turned into an evolutionary partial differential equation (PDE) on the function $\phi$.

The Chan-Vese algorithm is robust with respect to noise and as such it can be applied to medical images containing inevitable acquisition artefacts. Additionally, it can successfully segment images without large intensity gradients, i.e., without sharp edges. It is worth noting that the ChanVese algorithm can be extended to vector-valued images and to finding several disjoint regions at the same time.

\section{ACTIVE CONTOUR MODEL}

An active contour model (using level sets) is the optimisation technique used to find an unknown outline based on an initial contour and iterative procedures to optimise the zero level until the outline is found. Active contours and active surfaces are means of model driven segmentation. Their use enforces closed and smooth boundaries for each segment irrespective of the image content. The contour is defined in the $(\mathrm{x}, \mathrm{y})$ plane of an image as a parametric curve. Contour is said to possess an energy (Esnake) which is defined as the sum of the three energy terms. The energy terms are defined cleverly in a way such that the final position of the contour will have a minimum energy (Emin). Therefore our problem of detecting objects reduces to an energy minimization problem. ACM models treat segmentation as an energy minimization problem where the energy of an active spline/contour is minimized by PDEs-based methods toward the objects' boundaries. In classic ACMs, detecting objects' boundaries is by image gradients. However, this has one main drawback that it remain at a local minimum. Therefore, it cannot get adequate segmentation results. In the past two decades, a number of ACMs have been proposed, such as active contour without edge (ACWE) model and fast global minimization-based active contour model (FGM-ACM) proposed by Bresson et al. The ACWE model can be prepared as the following energy minimization problem:

$$
\begin{aligned}
\min _{\Omega_{c}, c_{1}, c_{2}} & \left\{E_{1}^{A C W E}\left(\Omega_{c}, c_{1}, c_{2}, \lambda\right)\right. \\
= & \int_{0}^{\operatorname{Length}(C)} d s \\
& +\lambda \int_{\Omega}\left(c_{1}-f(x)\right)^{2} d x \\
& \left.+\lambda \int_{\Omega / \Omega_{c}}\left(c_{2}-f(x)\right)^{2} d x\right\},
\end{aligned}
$$

where ds is the Euclidean element of length, the first term of Eq.(3) is the length of the curve $\mathrm{C}$, and $\mathrm{f}$ is the image to be segmented, $\Omega \mathrm{c}$ is a closed subset of the image f domain $\Omega$. The mean value of $f$ outside and inside are denoted as $\mathrm{c} 1$ and c2, respectively. $\lambda$ is an arbitrary fixed parameter $(\lambda>0)$ to controls the balance between regularization process and $c 1$, c2. The energy $\mathrm{E}_{1}{ }^{\mathrm{ACW} E}$ can improve because it naturally adds more constraints including the contour length than DC and CE loss function. In order to solve the segmentation formulation, Heaviside function of level set method and PDEs were introduced to decrease the energy $\mathrm{E}_{1}{ }^{\mathrm{ACW}} \mathrm{E} . \mathrm{E}_{1}{ }^{\mathrm{ACW}} \mathrm{E}$ can be rewritten as follows:

$$
\begin{aligned}
& E_{2}^{A C W E}\left(\Omega_{c}, c_{1}, c_{2}, \lambda\right) \\
& =\int_{\Omega}\left|\nabla H_{\epsilon}(\phi)\right| d x \\
& \left.\quad+\lambda \int_{\Omega} H_{\epsilon}(\phi)\left(c_{1}-f(x)\right)^{2} d x\right\} \\
& \left.\quad+\lambda \int_{\Omega / \Omega_{c}} H_{\epsilon}(-\phi)\left(c_{2}-f(x)\right)^{2} d x\right\}
\end{aligned}
$$

where $\mathrm{H}_{\epsilon}$ is a smooth approximation of the Heaviside function. And the gradient descent method minimizing of $\mathrm{E}_{2}{ }^{\mathrm{ACW} E}$ is defined as:

$$
\partial_{t} \phi=H_{\epsilon}^{\prime}(\phi)\left\{\operatorname{div}\left(\frac{\nabla \phi}{|\nabla \phi|}\right)-\lambda r_{1}\left(x, c_{1}, c_{2}\right)\right\}
$$

where $\mathrm{r} 1(\mathrm{x}, \mathrm{c} 1, \mathrm{c} 2)=(\mathrm{c} 1-\mathrm{f}(\mathrm{x})) 2-(\mathrm{c} 2-\mathrm{f}(\mathrm{x})) 2$ shown in $\mathrm{Eq}$ below. However, PDEs-based solutions including ACWE need to be solved on each individual image, which is time consuming. While they can give very good results, this makes ACWE less suitable for application in clinical settings where fast results, often as short as a few seconds, are needed. In order to achieve global minimization fast and stable, a EACW $\mathrm{E}$ based on total variation energy $\mathrm{T} \mathrm{V}$ was proposed [3] which is defined as in Eq.(6): 


$$
E_{4}^{A C W E}\left(u, c_{1}, c_{2}, \lambda\right)=T V_{g}(u)+\lambda \int_{\Omega} r_{1}\left(x, c_{1}, c_{2}\right) u d x
$$

where $\mathrm{u}$ is a characteristic function $1 \Omega \mathrm{c} . \mathrm{T} \mathrm{Vg}(\mathrm{u})$ is total variation energy. Eq above can also be written as:

$$
\begin{array}{r}
E_{4}^{A C W E}\left(u, c_{1}, c_{2}, \lambda\right)=\underbrace{\int_{0}^{\operatorname{Length}(C)} g|\nabla u| d s}_{\text {Length }} \\
+\lambda \underbrace{\int_{\Omega}\left(\left(c_{1}-f(x)\right)^{2}-\left(c_{2}-f(x)\right)^{2}\right) u d x}_{\text {Region }}
\end{array}
$$

where, $\mathrm{u}$ is a characteristic function valued between 0 and 1 . $\mathrm{E}_{4}{ }^{\mathrm{ACW}} \mathrm{E} \quad \mathrm{Eq}$ above provides a global minimum for ACWE model. Moreover, due to the limitation of the previous version of ACWE model based on Heaviside function and PDEs based solutions, it provides a fast and non-stationary solution while $\mathrm{u}$ is restricted from 0 and 1 . And also, this minimization problem of ACME to carry out segmentation task is able to apply into the deep learning field as it is constrained and some parameters can be fixed due to supervised learning and some parameters can be treated as trainable parameters to evaluate this minimization equation in an end-to-end learning fashion.

\section{RESULTS}

\section{Active Contour Function:}

In the current section, the test images are segmented based on their intensities alone. The first example here is the image of Zebra, which is considered to be relatively difficult to segment due to the multimodality of the pdf related to the object class. The segmentation results obtained for this image are shown in Fig, the four subplots of which depict the initial, two intermediate and the final shape of the active contour, respectively. The corresponding empirical densities of the object and background classes are shown in the lower row of subplots in Fig. We note that the initial position of the contour was chosen to be such that the number of data samples in both segmentation classes were equal. One can see that the algorithm results in a useful segmentation, which well agrees with the true shape of Zebra. It should be noted, however, that the final segmentation ascribes a portion of the shadow near Zebra's hoofs to the object class. It is because that the intensity levels of the shadow are very close to those of the stripes on Zebra's skin. In this case, the intensity information is insufficient to achieve the ideal result.
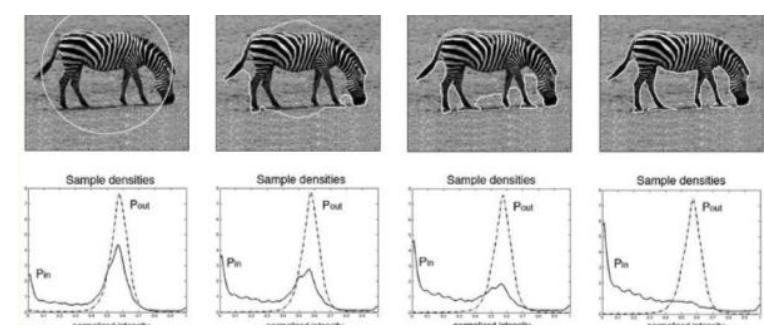

(Upper row of subplots) Intensity-based segmentation of Zebra. (Lower row of subplots) Corresponding empirical densities of Zebra and its background.

\section{Level Set Function:}

This model is based on the presumption that the intensity in each region can be approximated by a piecewise smooth function, therefore, it can handle some images with intensity Inhomogeneity. However, this model has a very expensive computational cost which limits its application in practice. So to overcome this paper proposed a new local region-based level set model in a vibrational level set formulation for image segmentation. Difficulties in practical applications arise due to the presence of noise, complex background, low intensity contrast with weak edges and intensity Inhomogeneity. This model, a data term with a local region-based function is introduced to stop the contours at edges. Then, the data term is incorporated into a vibrational level set framework with a length term to smooth the contour and a distance regularization term to maintain the evolution of contour stable. The initial contour with this model can be served as a constant function which is convenient and efficient. Results obtained on synthetic and medical images show good performance in handling with images with intensity Inhomogeneity and noise. The result of this method is as shown below.
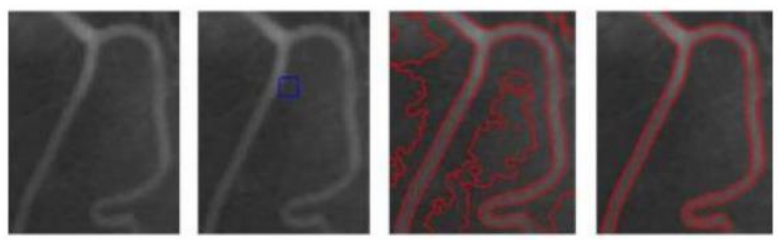

Segmentation of vessel image. (a)Vessel image (b)Initial contour (b)Intermediate

\section{CONCLUSION}

The main conclusion from this work is that there is no ideal segmentation method. Both parametric and geometric active contours are driven by forces extracted from the image itself, what makes them extremely dependent on the image quality, that is, lowly noised, fair definition of the structures' edges and absence of local minima. Even if one is able to overcome these problems, there are still further difficulties, like the initialization problem for example, which has a strong impact 
on the correct contour's convergence. This type of problem may result into the procedure to be repeated until the result obtained is good enough for the user.

\section{REFERENCE}

[1] B. Corona, M. Nakano, H. Pérez, (2004) "Adaptive Watermarking Algorithm for Binary Image Watermarks", Lecture Notes in Computer Science, Springer, pp. 207215.

[2] A. A. Reddy and B. N. Chatterji,( 2005) "A new wavelet based logo-watermarking scheme," Pattern Recognition Letters, vol. 26, pp. 1019-1027.

[3] P. S. Huang, C. S. Chiang, C. P. Chang, and T. M. Tu,( 2005) "Robust spatial watermarking technique for colour images via direct saturation adjustment," Vision, Image and Signal Processing, IEE Proceedings -, vol. 152, pp. 561-574,.

[4] F. Gonzalez and J. Hernandez, (1999)" A tutorial on Digital Watermarking ", In IEEE annual Carnahan conference on security technology, Spain,.

[5] D. Kunder,(2001) "Multi-resolution Digital Watermarking Algorithms and Implications for Multimedia Signals", Ph.D. thesis, university of Toronto, Canada,.

[6] J. Eggers, J. Su and B. Girod,(2000)" Robustness of a Blind Image Watermarking Scheme", Proc. IEEE Int. Conf. on Image Proc., Vancouver,.

[7] Barni M., Bartolini F., Piva A., (2002) Multichannel watermarking of color images, IEEE Transaction on Circuits and Systems of Video Technology 142-156.

[8] Kundur D., Hatzinakos D., (2004) Towards robust logo watermarking using multiresolution image fusion, IEEE Transcations on Multimedia 6 185-197.

[9] C.S. Lu, H.Y.M Liao, (2001) "Multipurpose watermarking for image authentication and protection," IEEE Transaction on Image Processing, vol. 10, pp. 1579-1592,.

[10] L. Ghouti, A. Bouridane, M.K. Ibrahim, and S. Boussakta,(2006) "Digital image watermarking using balanced multiwavelets", IEEE Trans. Signal Process., , Vol. 54, No. 4, pp. 1519-1536.

[11] P. Tay and J. Havlicek, (2002)"Image Watermarking Using Wavelets", in Proceedings of the IEEE, pp. II.258 - II.261,

[12] P. Kumswat, Ki. Attakitmongcol and A. Striaew,(2005) "A New Approach for Optimization in Image Watermarking by Using Genetic Algorithms", IEEE Transactions on Signal Processing, Vol. 53, No. 12, pp. 4707-4719, December.
[13] H. Daren, L. Jifuen,H. Jiwu, and L. Hongmei,( 2001) "A DWT-Based Image Watermarking Algorithm", in Proceedings of the IEEE International Conference on Multimedia and Expo, pp. 429-432,

[14] C. Hsu and J. Wu, August (1998) "Multi-resolution Watermarking for Digital Images", IEEE Transactions on Circuits and Systems- II, Vol. 45, No. 8, pp. 1097-1101.

[15] R. Mehul, (2003)"Discrete Wavelet Transform Based Multiple Watermarking Scheme", in Proceedings of the 2003 IEEE TENCON, pp. 935-938. 